\title{
Nonlinear Optics and Quantum Entanglement of Ultra-Slow Single Photons
}

\author{
M. D. Lukin ${ }^{1}$ and A. Imamoğlu ${ }^{2}$ \\ ${ }^{1}$ ITAMP, Harvard-Smithsonian Center for Astrophysics, Cambridge, Massachusetts 02138 \\ 2 Dept. of Electrical and Comp. Engineering, and Dept. of Physics, University of California, \\ Santa Barbara, CA 93106
}

(February 1, 2008)

\begin{abstract}
Two light pulses propagating with ultra-slow group velocities in a coherently prepared atomic gas exhibit dissipation-free nonlinear coupling of an unprecedented strength. This enables a single-photon pulse to coherently control or manipulate the quantum state of the other. Processes of this kind result in generation of entangled states of radiation field and open up new prospectives for quantum information processing.
\end{abstract}

PACS numbers 42.50.-p, 42.65.-k, 42.50.Dv,03.67.-a

Typeset using REVTEX 
It has been known for more than thirty years that light fields or photons can interact with each other in atomic media much like massive particles do [1]. However, the strength of the interaction of two single light quanta is typically extremely weak. As a result, conventional nonlinear optics is feasible only when powerful laser beams, containing a large number of photons, interact in nonlinear materials.

This Letter describes a method that allows for two slow light pulses [2 [4 of tiny energies to interact in a resonant ensemble of atoms. When Electromagnetically Induced Transparency (EIT) [5,6] is established for both of these pulses, they will propagate with ultra-slow but equal group velocities, and a very efficient nonlinear interaction between them will take place [7]. This interaction can be maintained for a very long time without dissipation, resulting in a new regime of quantum nonlinear optics. Specifically we describe here a scheme in which a traveling light pulse with an energy corresponding to that of a "single photon" $(\hbar \nu)$ can modify the refractive index of a second traveling pulse such that the latter experiences a non-linear phase shift on the order of $\pi$. This process allows one to create strongly correlated (i.e. entangled) states [5] of interacting photons and to generate macroscopic quantum superpositions such as Schrödinger cat states [8]. The technique described here opens up interesting prospects for coherent processing of quantum information 9 [11] in applications such as quantum computation and quantum communication.

It has been widely accepted that the use of ultra-high finesse micro-cavities is essential for having strong interactions between single photons 12 14. In the present contribution a radically different approach is taken, in that the properties of an optical material are "designed" to achieve an unprecedented strength of nonlinearity without the need for a cavity. Earlier work has already demonstrated that such a coherently controlled dense atomic ensemble displays remarkable phenomena [5,6], which are of great interest for nonlinear optics at a low light level [15 18].

We consider a process in which a pair of very weak optical fields $E_{1}, E_{2}$ interact resonantly with an ensemble of $N_{1}$ multi-state atoms (species A) as depicted in Fig.1. These atoms are coherently driven by a classical (i.e. many-photon) light field with a Rabi frequency $\Omega_{1}$ 
tuned to resonance with an atomic transition $\left|c_{1}\right\rangle \rightarrow\left|a_{1}\right\rangle$. We assume that the frequency of one of the weak optical fields $\left(E_{1}\right)$ is tuned to resonance with transition $\left|b_{1}\right\rangle \rightarrow\left|a_{1}\right\rangle$. Quantum interference induced by the driving field results in a sharp transmission resonance in the spectrum of the weak field $E_{1}$ (Fig. 2). The second weak field $\left(E_{2}\right)$ couples another optically allowed transition $\left|c_{1}\right\rangle \rightarrow\left|d_{1}\right\rangle$ with a single photon detuning $\Delta$. When $E_{2}$ is absent a properly tuned $E_{1}$ propagates without loss or refraction, but its group velocity is substantially reduced: this is the essence of EIT. The off-resonant field $E_{2}$ induces a Stark shift of the state $\left|c_{1}\right\rangle$ and therefore modifies the refractive index of the first field. Resulting phenomenon corresponds to cross-phase modulation of two weak light waves [7]: since the dispersion of refractive index is very steep (Fig.2), small Stark shifts result in a large index change.

The key idea of the present work is to arrange the conditions such that the two weak light pulses can interact in transparent, nonlinear media for a very long time. This is the case when pulses propagate with equal, slow group velocities. Photon interaction will then cause large nonlinear phase shifts even if pulses of ultra-small energies are involved. A possible technique to achieve equal group velocities for two weak pulses is explained in Fig.1: here $N_{2} \Lambda$-type atoms of a second kind (B) are added to the interaction region, and the frequency of the field $E_{2}$ is chosen to be on resonance with the optical transition $\left|b_{2}\right\rangle \rightarrow\left|a_{2}\right\rangle$. Application of a second driving classical field $\Omega_{2}$ of appropriate intensity will result in induced transparency and properly reduced group velocity for the field $E_{2}$. The mixture of atoms required to achieve such group velocity matching can be designed by using different isotopes of alkali atoms, and by applying an appropriate magnetic field to create the desired level splittings.

We describe quantum radiation by multi-mode field operators [5]: $\hat{E}_{1,2}(z, t)=$ $\sqrt{2 \pi c / L} \sum_{k} \hat{a}_{k 1,2}(t) e^{-i k z}$, where $\hat{a}_{k i}$ is the annihilation operator corresponding to the field mode with wave-vector $k$. $L$ is the quantization length and $c$ is the speed of light in vacuum. In the present approach we choose to restrict the quantization to a continuum of modes with wave-vectors ranging from $k_{0}-\Delta \omega / 2 c$ to $k_{0}+\Delta \omega / 2 c$, which in turn gives equal space-time 
commutation relations $\left[\hat{E}_{i}(z, t), \hat{E}_{j}^{+}(z, t)\right]=\Delta \omega \delta_{i j}$, that depend explicitly on the bandwidth of the system $\Delta \omega$. This bandwidth can be determined, e.g., by the detection process. As we will discuss shortly, the spectral width of the transparency window also gives a natural limitation on the maximal bandwidth.

The dynamics of the atomic media is described by Heisenberg equations for atomic polarizations and coherences. The finite bandwidth of quantized fields allows us to apply adiabatic elimination of the atomic degrees of freedom. By disregarding time derivatives of third and higher order, we arrive at the following evolution equations for the field operators:

$$
\begin{aligned}
\left(\frac{\partial}{\partial z}+\frac{1}{v_{g}} \frac{\partial}{\partial t}\right) \hat{E}_{1}=-\kappa \hat{E}_{1} & +\beta \frac{\partial^{2}}{\partial t^{2}} \hat{E}_{1} \\
& +i \eta \hat{E}_{2}^{+} \hat{E}_{2} \hat{E}_{1}+\hat{F}_{1} \\
\left(\frac{\partial}{\partial z}+\frac{1}{v_{g}} \frac{\partial}{\partial t}\right) \hat{E}_{2}=-\kappa \hat{E}_{2} & +\beta \frac{\partial^{2}}{\partial t^{2}} \hat{E}_{2} \\
& +i \eta \hat{E}_{1}^{+} \hat{E}_{1} \hat{E}_{2}+\hat{F}_{2} .
\end{aligned}
$$

The adiabatic expansion procedure used in derivation of Eqs. (1,2,2) is analogous to that of Refs. [15,17] for classical fields. Here, $v_{g}=c /\left(1+n_{g}\right)$ is the group velocity for the corresponding pulse, $n_{g}=g^{2} N /|\Omega|^{2}$, and $\kappa$ being the rate of residual single-photon loss $\kappa=n_{g} \gamma_{b c} / c . \quad \hat{F}_{1}$ and $\hat{F}_{2}$ are delta-correlated noise operators associated with dissipation. $\eta=\left[n_{g} g^{2} /\left(i \gamma_{c d}+\Delta\right)\right] l /\left(2 \pi c^{2}\right)$ is the rate of nonlinear interaction between pulses and $\beta=$ $n_{g} \gamma_{a b} /\left(|\Omega|^{2} c\right) . \quad g=\sqrt{\gamma_{a b} \sigma c /(2 A l)}$ is a normalized atom-field coupling constant, $A$ is the cross-sectional area of the quantized fields, $\sigma$ is resonant absorption cross-section and $l$ is the length of the interaction region. $\gamma_{i j}$ are linewidths of $|i\rangle \rightarrow|j\rangle$ atomic transitions. We assumed that all interacting atoms have identical linewidths and coupling constants, and that the number of atoms are equal $\left(N_{1}=N_{2}=N\right)$. To obtain equal group velocities, identical Rabi-frequencies for the driving fields were chosen $\left(\Omega_{1}=\Omega_{2}=\Omega\right)$.

Eqs. (11,2) together with the commutation relation define a quantum field theory of interacting pulses in a coherently prepared medium. In cases when losses can be neglected this field theory is analogous to that of a weakly interacting Bose-gas, but with an imaginary effective mass term. This imaginary mass term results from the finite bandwidth of the 
transparency window and causes a spreading of the input pulses (Fig.2).

We first consider the classical limit of this theory by replacing operators $\hat{E}_{1,2}$ with their expectation values $E_{1,2}$. In the ideal case $\Delta$ can be chosen to be large and hence $\eta$ is purely real. When attenuation and pulse spreading are small enough to be neglected, Eqs. (11,2) can be easily solved by $E_{1,2}(z, t)=E_{1,2}\left(0, t^{\prime}\right) \exp \left(i \eta\left|E_{2,1}\left(0, t^{\prime}\right)\right|^{2} z\right)$, which corresponds to cross-phase modulation of the weak beams. Note that this result is expressed in terms of retarded time $t^{\prime}=t-z / v_{g}$. Magnitude of the nonlinear phase shift at the peak of the pulse can be expressed in terms of energy $E$ and duration $T$ of a Gaussian pulse:

$$
\text { phase shift }=\sqrt{\frac{\ln (2)}{4 \pi}} \frac{\gamma_{c d}}{\Delta} \frac{\sigma}{A} \frac{E}{\hbar \nu} \frac{\tau_{g}}{T} .
$$

Here nonlinearity $\eta$ is expressed in terms of group delay $\tau_{g}=n_{g} z / c$. In the limit when $v_{g} \ll c$ the later corresponds to the interaction time of the pulse with the medium.

It is apparent that large phase shifts exceeding $\pi$ at energies corresponding to a fraction of $\hbar \nu$ appear to be possible when the interaction time exceeds the pulse duration of a tightly focused $(\sigma \sim A)$ laser beam. Before proceeding it is important to re-examine the assumptions resulting in this striking conclusion.

First, we note that a stringent limitation imposed by the difference of group velocities of interacting pulses [17] is absent in our case since the interaction is designed such that group velocities are equal. Second, even in the absence of various absorption mechanisms, nonlinear phase shifts are limited by the bandwidth of the transparency window which decreases with propagation distance [19] $\Delta \omega_{\max }=(\beta z)^{-1 / 2}$. After a sufficiently long propagation this results in spreading of the pulse (see Fig.2). In order to avoid losses due to spreading, $\tau_{g} / T$ should be smaller than

$$
\tau_{g} \Delta \omega_{\max }=\sqrt{\frac{\Omega^{2} \tau_{g}}{\gamma_{a b}}}=\sqrt{\frac{\sigma N}{2 A}} .
$$

The quantity under square root corresponds to an optical depth of the medium [19]. Hence it is essential to operate in optically dense gas. Finally we note that the interaction time $\left(\tau_{g}\right)$ is itself limited due to absorption associated with decoherence of the "dark state" [6]; i.e. 
$\tau_{g}<\gamma_{b c}^{-1}$ must be satisfied. This implies that EIT resonances should be strongly saturated, i.e. $\Omega^{2} / \gamma_{a b} \gamma_{b c} \geq N \sigma / A \gg 1$, in order for nonlinear phase shifts to be large.

We now consider quantum dynamics of the nonlinear interaction. When absorption is negligible and the bandwidth $\delta$ of the pulses satisfy $\delta<\Delta \omega<\Delta \omega_{\max }$, quantum Eqs. (12,2) can be solved by

$$
\hat{E}_{1,2}(z, t)=\hat{E}_{1,2}\left(t^{\prime}\right) \exp \left[i \eta \hat{E}_{2,1}^{+}\left(t^{\prime}\right) \hat{E}_{2,1}\left(t^{\prime}\right) l\right]
$$

where $\hat{E}_{i}(t)$ are Heisenberg operators describing the input fields at $z=0$. We first analyze the evolution of most "classical" of all possible input wave packets. These are multi-mode coherent states [5] $\left|\alpha_{1}, \alpha_{2}\right\rangle=\prod_{k, j}\left|\alpha_{1}^{k}\right\rangle \times\left|\alpha_{2}^{j}\right\rangle$, which are eigenstates of input operators $\hat{E}_{i}(t)$ with eigenvalues (at $z=0) \alpha_{i}(t)=\sqrt{2 \pi c / L} \sum_{k} \alpha_{i}^{k} e^{i k c t}$. After propagation through the nonlinear dispersive medium, the following expectation values of the fields can be measured:

$$
\begin{aligned}
& \left\langle\hat{E}_{1,2}(z, t)\right\rangle=\alpha_{1,2}\left(t^{\prime}\right) \times \\
& \exp \left[\left[-2 \sin ^{2}(\Phi / 2)+i \sin (\Phi)\right] \frac{\left|\alpha_{2,1}\left(t^{\prime}\right)\right|^{2}}{\Delta \omega}\right],
\end{aligned}
$$

where quantum phase shift $\Phi=\left[1 /(4 \pi) \sigma / A \gamma_{c d} / \Delta\right] \Delta \omega \tau_{g}$. These solutions have the same form as those obtained in Ref. [20] for single-mode fields and generalize that earlier result to the multi-mode case that is appropriate for the traveling-wave geometry.

Eq. (6) reproduces the classical result only when $\Phi \rightarrow 0$; for sufficiently large interaction times the quantum dynamics of wave packets deviates substantially from the classical case. In particular, both phases and amplitudes given by Eq. (6) exhibit periodic collapses and revivals. The origin of this behavior can be understood by noting that each component of input coherent states $\left|\alpha_{1}^{k}\right\rangle\left|\alpha_{2}^{j}\right\rangle$ is itself a coherent superposition of many Fock components $\left|n_{1}^{k} m_{2}^{j}\right\rangle$. During nonlinear interaction each of these components acquires a different phase change resulting, for sufficiently large $\Phi$, in quantum dephasing of the original coherent states. However when $\Phi$ reaches multiples of $2 \pi$, all components "re-phase" such that original coherent state is reproduced. At intermediate values of interaction times, re-phasing to other macroscopic states can occur. For example, when $\Phi=\pi$ the output state of two 
fields can be verified to be: $|\psi\rangle=\frac{1}{2}\left(\left|\alpha_{1}, \alpha_{2}\right\rangle+\left|-\alpha_{1}, \alpha_{2}\right\rangle+\left|\alpha_{1},-\alpha_{2}\right\rangle-\left|-\alpha_{1},-\alpha_{2}\right\rangle\right)$. This is an entangled superposition of macroscopically distinguishable states. Superpositions of this kind have no classical counter-part, and correspond to Schrödinger cat-like states [8].

Consider now a different type of input quantum state corresponding to multi-mode singlephoton wave-packets: $\left|1_{i}\right\rangle=\sum_{k} \xi_{k} \hat{a}_{k, i}^{\dagger}|0\rangle$ where Fourier amplitudes $\xi_{k}$ are normalized such that $\sum_{k}\left|\xi_{k}\right|^{2}=1$. In free space these single-photon states represent traveling waves initially $(t=0)$ localized around $z=0$. The dynamics of individual wavepackets is fully described by a single photon "wave-function" $\tilde{\Psi}_{i}(t, z)=\left\langle 0\left|\hat{E}_{i}(t)\right| 1_{i}\right\rangle$. Correlations of these wavepackets emerging due to photon-photon interaction are described, in turn, by correlation amplitudes [5] $\tilde{\Psi}_{12}\left(t_{1}, z_{1} ; t_{2}, z_{2}\right)=\left\langle 0\left|\hat{E}_{1}\left(t_{1}, z_{1}\right) \hat{E}_{2}\left(t_{2}, z_{2}\right)\right| 1_{1} 1_{2}\right\rangle$. After propagation though the atomic cell the correlation amplitude is given by:

$$
\begin{aligned}
\tilde{\Psi}_{12}\left(t_{1}^{\prime \prime}, z_{1}\right. & \left.; t_{2}^{\prime \prime}, z_{2}\right)=\tilde{\Psi}_{1}\left(0, t_{1}^{\prime \prime}\right) \tilde{\Psi}_{2}\left(0, t_{2}^{\prime \prime}\right) \\
& \times\left[1+\operatorname{sinc}\left[\Delta \omega\left(t_{1}^{\prime \prime}-t_{2}^{\prime \prime}\right) / 2\right]\left(e^{i \Phi}-1\right)\right],
\end{aligned}
$$

where $t_{i}^{\prime \prime}=t_{i}-l / v_{g}-\left(z_{i}-l\right) / c$. Eq. (7) indicates that the nonlinear interaction alters the mode structure of the pulse in addition to generating a phase shift on existing components. This phenomenon is related to the classical effect of pulse broadening due to cross-phase modulation. It is important however that equal-time ("coincidence") correlations ( $t_{1}^{\prime \prime}-$ $\left.t_{2}^{\prime \prime}\right) \Delta \omega \ll 1$ indicate that a pair of single photons acquire a phase shift $\Phi$ as a result of the nonlinear interaction. The magnitude of $\Phi$ can easily exceed $\pi$, when $\tau_{g} \Delta \omega_{\max } \gg 1$.

These large nonlinear phase shifts can be used to create quantum entanglement. For example, if the photons are initially in coherent superpositions of two states (for example the two polarization states $\left.\left|1_{ \pm}\right\rangle\right)$, and only one of these states $\left(\left|1_{+}\right\rangle\right)$is subject to the strong nonlinear interaction, the resulting state cannot be factorized into a product state of individual modes [21]. Operations of this kind form the essence of quantum information processing [10]. The example considered above indicates, however, that the present approach to quantum entanglement differs conceptually from the techniques discussed previously. In particular, usual approaches to quantum processing are based on systems whose Hilbert space can be 
restricted to a 2 dimensional subspace (qubit). In contrast, the present technique involves multi-mode, traveling wave excitations where the 2-dimensional Hilbert space of the qubit (i.e. polarization) and the external degrees of freedom of the photon field become coupled. It is interesting to consider how various concepts of quantum information theory can be applied in the context of our approach.

It is clear that the feasibility of quantum entanglement depends upon the large delaybandwidth products $\Delta \omega_{\max } \tau_{g}$ corresponding to ultra-slow pulses. In experiments involving ultra-cold [2] and hot [19] atoms values of this product on the order of few tens have been observed. Potentially, an increase by two to three orders of magnitude is likely, which should allow for a high fidelity of entanglement. We conclude by noting that exceptionally large nonlinearities have already been measured in various experiments [2, 3, 19. In particular, an efficient nonlinear phenomenon corresponding to $\pi$ phase shift has been observed with pulse energies corresponding to less than $10^{3}$ photons per atomic cross section [22].

We thank Steve Harris for his encouragements and acknowledge stimulating discussions with him, M. Fleischhauer, R. Glauber, M. O. Scully, M. Werner and R. Walsworth. This work was supported by the National Science Foundation, a David and Lucile Packard Fellowship, and the US Army Research Office. 


\section{REFERENCES}

[1] N. Bloembergen, Nonlinear Optics (W.A.Benjamin, New York, 1965).

[2] L. V. Hau, S. E. Harris, Z. Dutton, and C. H. Behroozi, Nature 397, 594 (1999).

[3] M. M. Kash et al., Phys. Rev. Lett. 82, 5229 (1999).

[4] D. Budker et al., Phys. Rev. Lett. 83, 1767 (1999).

[5] M. O. Scully and M. S. Zubairy, "Quantum Optics”, Cambridge University Press, Cambridge 1997.

[6] S. E. Harris, Physics Today, 50, 36 (July, 1997).

[7] H. Schmidt, A. Imamoğlu, Opt. Lett., 21, 1936 (1996).

[8] E. Schrödinger, Naturwissenschaften 23, 812 (1935); Letter from Einstein to Schrödinger of 22 December 1950, in Briefe zur Wellenmechanik, edited by K.Przibram (Springer, Vienna, 1963 ), p.36.

[9] A. Ekert, and R. Jozsa, Rev. Mod. Phys. 68, 733-753 (1996).

[10] A. Steane, Rept. Prog. Phys. 61, 117 (1998).

[11] S. Lloyd, Sci.Am. 273, 140 (1995).

[12] H.J.Kimble, Physica Scripta, 76, 127 (1998).

[13] A. Imamoğlu, H. Schmidt, G. Woods, and M. Deutsch, Phys. Rev. Lett. 79, 1467 (1997).

[14] M. Dunstan, S. Rebic, S. Tan, S. Parkins, M. Collett, and D. Walls, in Proceedings: Quantum Communication, Computing, and Measurement 2. (ed. by P. Kumar, G. M. D'Ariano, and O. Hirota).

[15] S. E. Harris, Y. Yamamoto, Phys. Rev. Lett. 81, 3611 (1998).

[16] M. D. Lukin, A. Matsko, M. Fleischhauer, M. O. Scully, Phys. Rev. Lett. 82, 1847 
(1999).

[17] S. E. Harris and L. V. Hau, Phys. Rev. Lett. 82, 4611 (1999).

[18] See also J. D. Franson, T. B. Pittman, Phys.Rev.A, 60, 917 (1999).

[19] M. D. Lukin, et al., Phys. Rev. Lett. 79, 2959 (1997).

[20] B. C. Sanders, G. J. Milburn, Phys. Rev. A 45, 1919 (1992).

[21] G. J. Milburn, Phys. Rev. Lett. 62, 2124 (1989).

[22] A. S. Zibrov, M. D. Lukin, and M. O. Scully, "Non-degenerate Parametric SelfOscillation via Resonantly Enhanced Multi-wave Mixing in Coherent Atomic Media", Phys.Rev.Lett. 83, (1999), in press. 


\section{FIGURES}

FIG. 1. Prototype composite atomic medium for strong nonlinear interactions at the single-photon level. By adjusting detunings such that the two weak fields $E_{1}, E_{2}$ and two classical fields $\Omega_{1}, \Omega_{2}$, are in resonance with transitions in atoms $\mathrm{A}$ and $\mathrm{B}$ as shown, resonant absorption can be eliminated and the group velocities of two weak pulses can be made equal. The scheme can be implemented, for example, using a natural mixture of atomic rubidium. It consists of two isotopes $\mathrm{Rb}^{87}$ (natural abundance $\sim 25 \%$ ) and $\mathrm{Rb}^{85}(\sim 75 \%)$ with transition frequencies differing due to isotope shifts and nuclear spins. Propagation of tightly focused beams over long distance can be achieved using non-diffracting beams of Bessel shape, fiber waveguides, or focusing properties associated with EIT to induce waveguiding optically.

FIG. 2. (a) Spectrum of transmission and refractive index corresponding to EIT. Quantum interference in the atomic ensemble induced by a coherent driving field creates a sharp resonance in the transmission of a weak optical field, accompanied by rapid variation of the refractive index (red curve). This rapid variation causes a dramatic reduction of group velocity. The presence of a second weak field causes an effective shift of the resonant frequency (green curve), which results

in corresponding change of a refractive index and hence the phase of the first weak field. (b) Propagation dynamics in coherent media: when resonant pulses enter the medium, they exhibit spatial compression and very soon slip behind the reference pulse (white) that does not interact with the atoms. The total time that two slow pulses can spend in the medium is limited by the residual single photon loss, and by the spreading of the pulses after $\tau_{s} \sim \Omega^{2} T^{2} / \gamma_{a b}$. The spreading is due to a finite bandwidth $\Delta \omega_{\max }$ of the EIT resonance. 


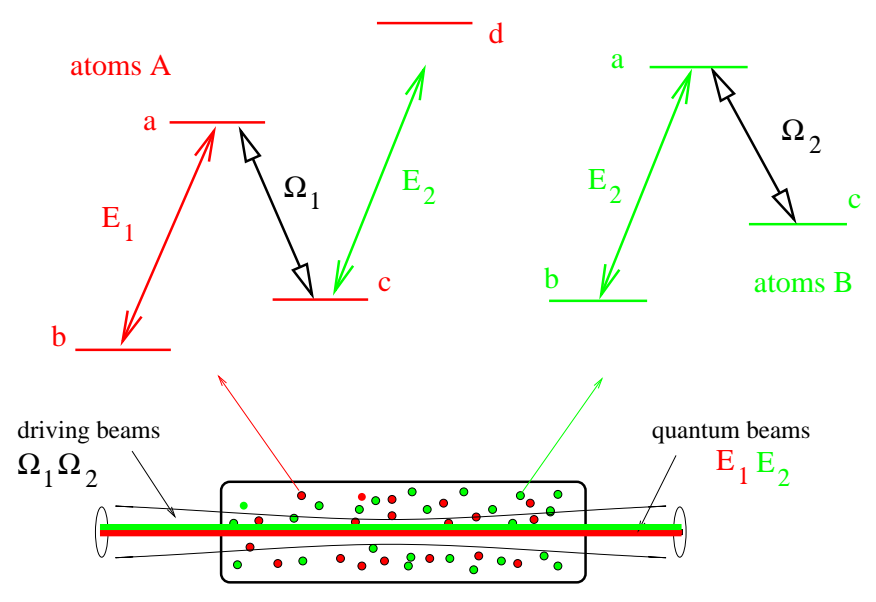

Figure 1 

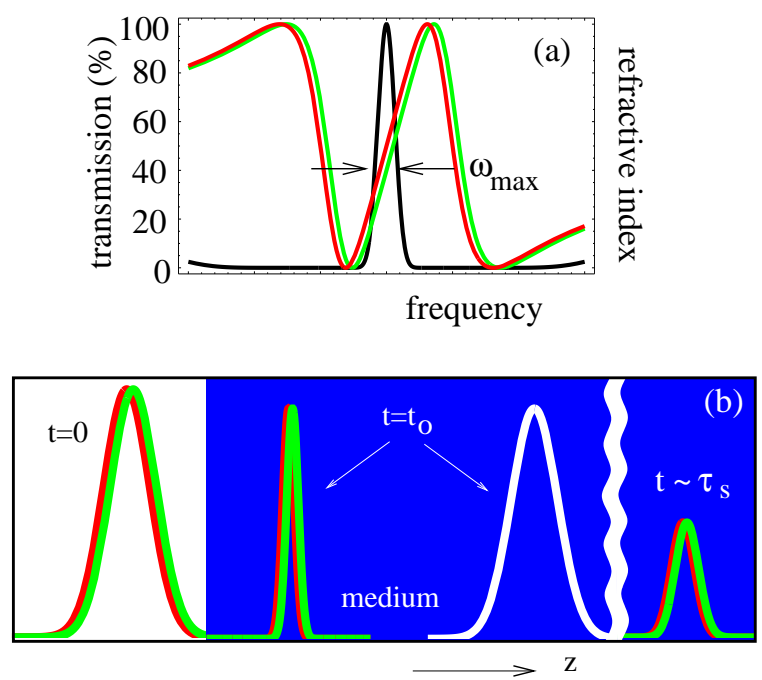

Figure 2 